\title{
EDITORIAL
}

\section{Invitation to scientific writing}

\section{Alina Chiracu}

The scientific world is complex and cruel. Scientific writing is difficult and chronophagous. However, the opportunities to enter this world and the awareness of the real contribution that we can bring to scientific knowledge bring with them the feeling of pride and the belief that we also have something to say. Moreover, we can bring into discussion the so special psychism of the Romanian people.

What does actually mean quality scientific writing? In a random order, here are some answers to this question.

It means keeping in mind the audience we are addressing. The target audience can be made up of experts - who are familiar with the topic, specialists - from different scientific fields, but who easily understand the scientific concepts we refer to, students - who do not have a high level of knowledge but who want to learn from what they read. Depending on the target audience, the writing will be more or less rigorous, formal, detailed.

It means to bring a note of originality, to expose our opinion, to present our results, to do what has not been done, to find what has not been found. Through scientific ethics and the authenticity of writing, we are original. Through the groups we choose for research and the combination of intruments we use, we are original again. By collecting specific data and associating them we are original again. How difficult can it be to be original?

It means to be precise and accurate. To convey the scientific information in an easy-to-understand form, to use simple but comprehensive words, to avoid metaphors and redundant expressions.

It means positioning us in the context of other scientific works because science is developing on the fundamentals already built by other specialists. On the one hand, the existing context is the starting point and the reason we write, and on the other hand is the end point of the research, aligning our own results with those obtained by other specialists. Explaining the existing context first shows that we have a certain level of knowledge and that we have gained the right to enter into the "conversation" of scientists, and then it helps integrate our results, align them with previous studies, associate with or dissociating from the results obtained in other cultures and other contexts.

It means to emphasize the pragmatic relevance of the research effort. How good is this effort? Who will benefit from our results? What benefits do we bring to humanity through what we have discovered? Writing words and figures does not mean to add value to the scientific knowledge. The scientific world from which we want to be part works collaboratively to deepen knowledge, to decipher the mysteries of human psychism, to elucidate the most complex equation that is the human being.

It means to be impartial, not to make unscientific suppositions, not to distort the meaning of the results, not to make inappropriate inferences. It is to schematize our research effort and make it known to those interested in knowing, learning or comparing.

It means to build a logical and meaningful structure, to respect the chronology of facts and ideas, to take into account the specific requirements of the publishers.

It means especially to have the courage to research, to look for the truth, to portray human psychism and to provide valuable information to 
readers, to complement the present scientific knowledge.

It means to involve our body and soul in the research activity and to believe that we can make our contribution in our field of expertise.

It means to work for science and for human kind. Unlike scientific writing, creative writing is limitless, fiction, non-fiction, or a combination of these. It is not addressed to a particular category of readers, nor does it necessarily intend to educate or teach someone. If scientific writing can be creative, through the novelty it brings, creative writing may rarely be scientific. While scientific writing uses a specialized language, creative writing uses different stylistic methods, epithets, metaphors, hyperboles. While scientific writing intends to describe an exact reality, creative writing imagines fictional realities and juggles with their destinies. If scientific writing is impersonal, organized and somewhat standardized, following a predefined logic pattern, creative writing has free boundaries, authors can make assertions and inferences, can relate interesting experiences, express their opinions, ideas or emotions. If scientific writing is objective, creative writing is subjective. If scientific writing stimulates the cognitive sphere, creative writing stimulates the affective sphere.

Scientific writing needs precise space and time elements, prior documentation, deadlines, confirmation, while creative writing can expand indefinitely, words can be freely placed on the page without the fear of eluding the great scientific truths, without fear of exceeding deadlines.

If some of us are inclined to creative writing, others are oriented towards scientific writing. If some of us are imaginative, others are realistic and pragmatic. If we meet all these conditions and if we want to contribute to universal knowledge, let's write. 